\title{
A theoretical approach to the adsorption of ions on metal surfaces
}

\author{
J.A.N.F. Gomes* ${ }^{*}$ Anna Ignaczak ${ }^{1}$ \\ CEQUP/Faculdade de Ciências, Universidade do Porto, Rua do Campo Alegre 687, 4150 Porto, Portugal
}

\begin{abstract}
This paper gives a short review of the current status of quantum and simulation studies of the specific adsorption phenomenon. In the light of results of some recent investigations on the adsorption of halide ions on noble metals, the difficult problem of the interfacial interactions is discussed. Several difficulties associated with the modeling of the adsorption process in the simulations are pointed out. Some of them are shown to be related to the cluster model calculations commonly used in the fitting of the analytical potentials for the ion-metal interaction. The adequacy of the additive analytical form of this potential which is usually applied, is also discussed. The problem of the description of the interaction of the ion with the electrode in the intermediate region, when the ion is still surrounded by water molecules, is also addressed. (C) 1999 Elsevier Science B.V. All rights reserved.
\end{abstract}

Keywords: Copper; Halide ions; Interfacial interactions; Potential of mean force; Specific adsorption

\section{Introduction}

The understanding of the structural properties of electrolytes near to the metal surface is of primary importance in electrochemistry. It is widely recognized that the synergism of experiment and computer simulations may be crucial to the progress in this understanding. The goal of this paper is to highlight some of the important progress achieved in the last decade.

In 1987, in a meeting dedicated to recent developments in double layer theory, S.L. Carnie stated the following: "While no simulations have yet been reported of water near a metal surface, the day cannot be far away" [1]. This statement was based on the progress observed in the development of (classic)

\footnotetext{
* Corresponding author.

E-mail address: jfgomes@fc.up.pt (J.A.N.F. Gomes)

${ }^{1}$ Present address: University of Lodz, Dept. of Theoretical Chemistry, ul. Pomorska 149/153, 90-236 Lodz, Poland.
}

pair potentials in the 1970s and 1980s and their application in describing the structural, thermodynamic and dynamic properties of liquid (bulk) water.

Since this time, great progress has been made in this area. The Monte Carlo (MC) method as well as Molecular Dynamics (MD) have been successfully used in the last decade for the investigation of the properties of the metal-water [1-16] and the metal-ionic solution interface [17-31]. A strategy commonly used for the description of interactions between all species involved in the simulated process, is to model them by some simple analytical functions derived from the results of quantum calculations. This methodology, being the standard way of construction of the ionwater potentials, has a relatively short history in the area of the interfacial interactions. The first quantum derived metal-water potentials were applied only around 1989 [7, 9] and the metal-ion potential only in 1991 [18]. Among the electrode metals, platinum has been the most extensively studied. In most reported results the first layer of water was found to 
be tightly bound to platinum atoms with infrequent excursions far from the preferred positions. In several works it was found that the structure of water near the metal surface resembles that of ice, but other arrangements of water molecules over the surface, mostly regular, have been also reported. From the standard MD simulations, the ions $\mathrm{Li}^{+}$and $\mathrm{I}^{-}$were found [18] to take positions in the surface away from their preferred positions when in a vacuum, this being above the hollow site of the metal surface. The small $\mathrm{Li}^{+}$has a strong interaction with water which prevails over the $\mathrm{Li}^{+}$-metal interaction. It is kept in the first solvation shell, at a distance from the surface much larger than that taken in the absence of waters. It should not be said to be contact adsorbed although no water molecule exist in between the ion and the metal. In fact, the preferred octahedral solvation cage around $\mathrm{Li}^{+}$loses about one water near the surface and this small ion fits in the first water layer without much distortion. The position taken by iodide, on the other hand, reflects its bigger size. It was found to occupy a position between the hollow and the bridge sites of the $\operatorname{Pt}(100)$ surface while the two neighbouring metal atoms lose their covering water molecules.

In another approach to the problem of specific adsorption of ions on metals [24-27], authors go much further in the discussion of the energetics. The way of the ion to the electrode surface is analyzed from the point of view of the solvent contribution to the free energy curves of the ions as a function of their distance from the metal wall. In the pioneering work of Rose and Benjamin [24] on $\mathrm{Na}^{+} \mathrm{Cl}^{-}$at the water$\mathrm{Pt}(100)$ interface it was shown that these solvent mean force curves do have some sort of local minimum in the region statistically preferred by the ions at the first solvation layer of the metal. However, the total free energy curves presented in that work are unrealistic as the ion-metal interaction was represented by the classical image charge potential that is recognized as being "...somewhat ambiguous and (...) not well defined for distances close to the metal'. In some more recent works [25-27], the free energy of adsorption was obtained using more sophisticated models of the ion-metal interaction, derived from the quantum calculations. The results for adsorption of halide ions on mercury [27] appeared to disagree with the common electrochemical wisdom.

The present paper reviews some very recent work going on in our laboratory on the structure and energetics of the solvation of halide ions at infinite dilution near a copper surface. The adsorption of ions on noble metals has been extensively investigated experimentally in the past [32-55]. Copper is an especially interesting metal for the theoretical studies, since its great tendency for oxidation strongly perturbs the experimental measurements. In this work several problems and some still open questions associated with the simulation studies of the interfacial processes will be pointed out.

\section{Specific adsorption of halide ions on noble metals in the light of our recent studies}

The current understanding of many aspects of the electrode-electrolyte systems depends on the interpretation of experimental results supported by continuum electrostatic models or simplified statistical mechanical calculations. It is normally assumed that the behaviour of an ion near the electrode depends on the competition between the ion-metal attraction and the needed desolvation of the ion as it approaches the surface due to the limited accessability of the water when the ion is near the wall. This qualitative idea is backed by phenomenological models but a molecularlevel treatment is lacking.

The present authors have considered the interactions of the halide ions [56, 57] and of the water particle [58] with metal surfaces by quantum calculations. From DFT cluster model calculations it has been found that the adsorption of water on the $\mathrm{M}(100)$ surface, where $\mathrm{M}=\mathrm{Cu}, \mathrm{Ag}, \mathrm{Au}$, occurs through the oxygen end of the $\mathrm{H}_{2} \mathrm{O}$ molecule and is characterized by a very low barrier for the migration between the bridge site and the top site, while the third position studied, the hollow site, has been found to be energetically forbidden for the adsorption of water. The adsorption of the water monomer at the top site was found to be stabilized when there is some tilt angle between the dipole moment of water and the normal to the surface. These cluster model calculations predict that for copper and gold the adsorption of the $\mathrm{H}_{2} \mathrm{O}$ monomer is stabilized at the bridge site, while for silver the top-tiled position is preferred. However, the adsorption energies at the bridge site and at the 

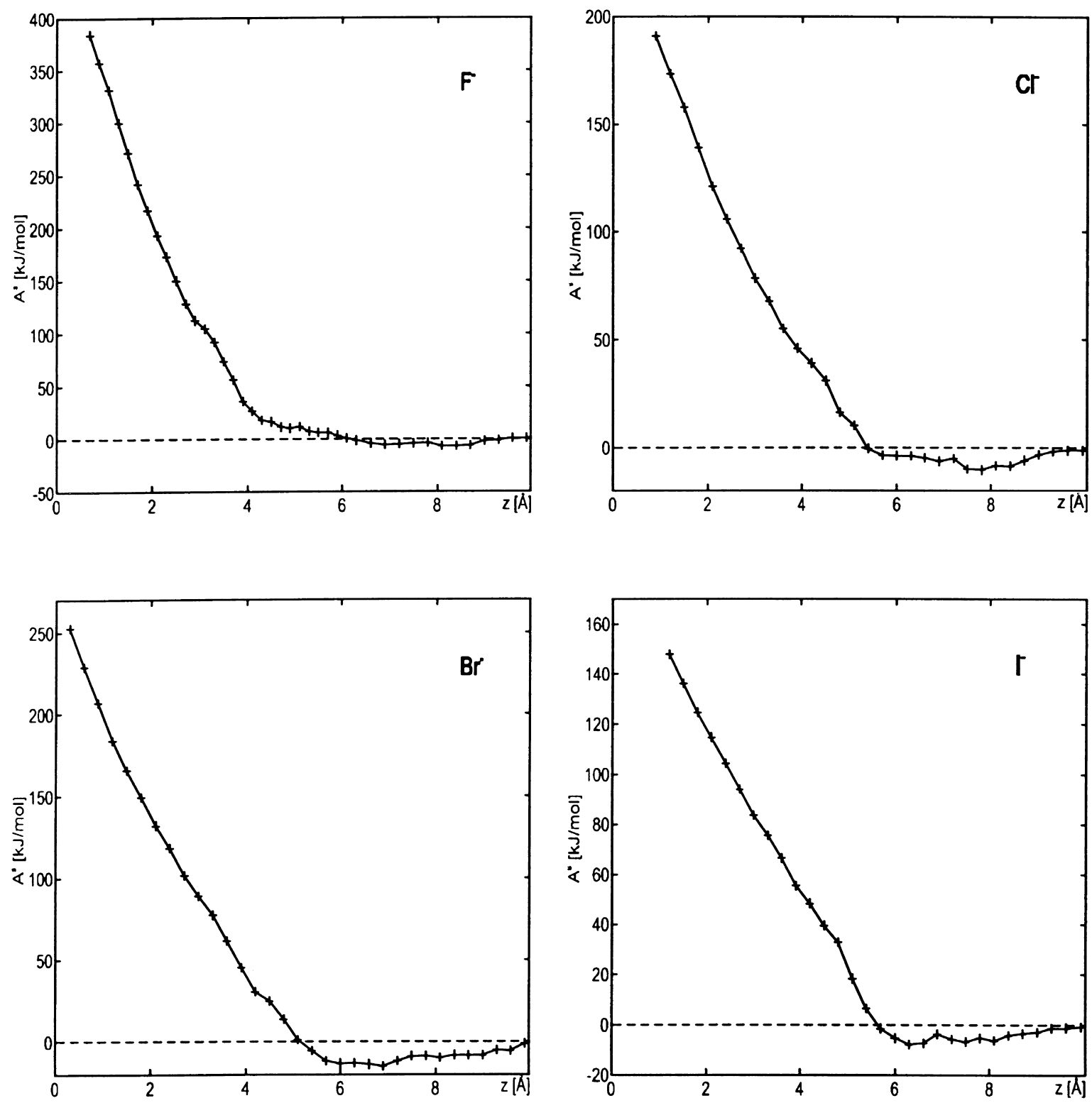

Fig. 1. The solvent mean force $A^{\mathrm{s}}$ on each of the four halide ions approaching the $\mathrm{Cu}(100)$ surface from the bulk solution.

top-tiled conformation do differ by no more than $1 \mathrm{~kJ} \mathrm{~mol}^{-1}$ thus being essentially indistinguishable.

The strength of the adsorption of water on noble metals has been found to be rather weak, in agreement with experimental estimates of this property $[59,60]$. The trend of hydrophilicity of the three metals has been found to be in the direction $\mathrm{Cu}>\mathrm{Au}>\mathrm{Ag}$.

The specific adsorption of ions has been studied from the point of view of the direct ion-metal interaction as obtained from the DFT cluster calculations [56,57]. The studies on this subject are especially important since there is no experimental estimates of the strength of this perhaps decisive force. There is also a very limited number of theoretical studies of this type [61-75], and the results obtained frequently exhibit unexpected features, in disagreement with the 
experimental knowledge. From the experimental point of view there is a lot of data on the structural characteristics of adsorption of ions on metals [41$48]$ giving evidence of the preferential adsorption of ions at the multifold hollow site of the surface. Nevertheless, no even approximated prediction could be made about the adsorption energy of the ion on metal.

The present authors found a clear tendency in the interaction energies of halide ions with noble metals. Unambiguously, the hollow site was found to be preferred for adsorption of ions on all three metals, followed by the bridge site and the least favoured top site. As suggested in some earlier quantum calculations on this subject [70-75], the tendency of adsorption of ions on a metal in vacuum has been found to be as follows: $\mathrm{F}^{-}>\mathrm{Cl}^{-}>\mathrm{Br}^{-}>\mathrm{I}^{-}$. This result is especially intriguing taking into account that the opposite order was reported from experiment: iodide has been found to be the strongest adsorbed on metals from aqueous solution, and fluoride the weakest [3238]. At the same time, for the three metals tested, gold appears to be the most attractive metal for the adsorption of halides, in agreement with experimental reports [32].

Considering the trend of the ion-water interaction that decreases in the same direction as the ion-metal interaction, one must realize that no final conclusions about the nature of the specific adsorption phenomenon can be taken on the basis of the quantum calculations. In the light of the quantum results, the particular balance of forces occurring near the surface requires more complex studies, using a method that allows one to consider all relevant interactions in their equilibrium state. A Monte Carlo (MC) simulation was adopted to perform the comparative studies of the specific adsorption of the halide ions on the $\mathrm{Cu}(100)$ surface in aqueous solution [76].

Typically, a rectangular prism of water is considered between two metal surfaces, the periodic boundary conditions being applied in the two directions parallel to the metal. When the two metal surfaces are equal, equilibrium properties should be symmetric about the central plane and statistical significance may be improved by averaging between the left and the right half cells. The number of water molecules considered in the cell is such as to give the normal density of $1 \mathrm{~g} \mathrm{~cm}^{-3}$ for bulk water in the central part of the box. This is normally achieved by trial and error as the exact volume available for water is difficult to predict. In fact, the water is excluded from the immediate vicinity of the metal surface due to its repulsive forces.

This is the method used in the simulation of the specific adsorption phenomenon. A lamina of 255 waters was located between two $\mathrm{Cu}(100)$ surfaces modeled by the $300 \mathrm{Cu}$ atoms distributed according to the fcc crystallographic structure. The number of waters between the walls has been chosen by performing several tests with various numbers of waters (or different separation of walls keeping the same number of waters). The separation of the walls giving the desired density of waters in the middle of the box has been established to be of $24.4 \AA$.

Two levels of investigation were carried out. First, from the series of MC simulations performed for each ion at different distances from the surface, the solvent contribution, $A^{\mathrm{s}}$, to the potential of mean force acting on the ion is computed using the free energy perturbation method. This allows one to analyze the path of the ion approaching the surface from the point of view of the response of the solvent only. The solvent mean force on the ion, $A^{\mathrm{s}}$, obtained from those simulations is shown in Fig. 1 for each ion separately. As can be seen, at shorter distances from the surface the solvent contribution to the mean force has an increasing repulsive character. Unlike that found in the study of Spohr [27], this repulsion follows the same tendency as the strength of the ion-water interaction, the largest being for fluoride and the smallest for iodide.

The advantage of the separate calculations of the solvent mean force, $A^{\mathrm{s}}$, is that, unlike standard simulations, the results obtained are independent of the description of the ion-metal interaction. This latter component is added to the solvent mean force only a posteriori, what allows the combination of the main result, $A^{\mathrm{s}}$, with different models of the ion-metal potential.

\section{Some methodological aspects}

One might ask why the separation of the two components, the $A^{\mathrm{s}}$ and the direct ion-metal interaction, is so important. Having the results of the quantum calculations one should be able to construct the ion-metal interaction potential that would 


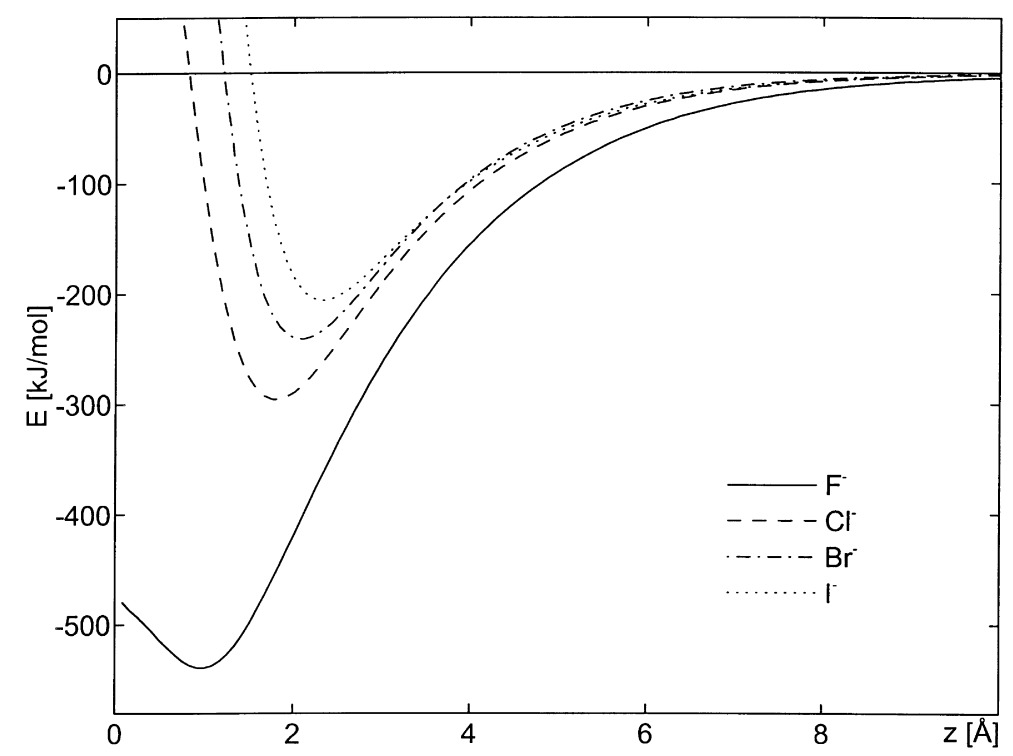

Fig. 2. The behaviour of the additive potential, $V_{1}$, when the ions are adsorbed at the hollow site of the $\mathrm{Cu}(100)$ surface.

unambiguously reproduce results of the cluster calculations. However, the problem is not that simple. The cluster model calculations introduce by their very nature the doubt of whether the small aggregate of atoms should be considered a sufficient approximation of the metal wall, or only a very limited representation of this wall. Is it correct to use a pairwise additive potential for this type of interaction? As has been shown in [76], where different models of the ionmetal potential have been tested, the final result in terms of the curve of the free energy of adsorption is largely determined by the ion-metal component.

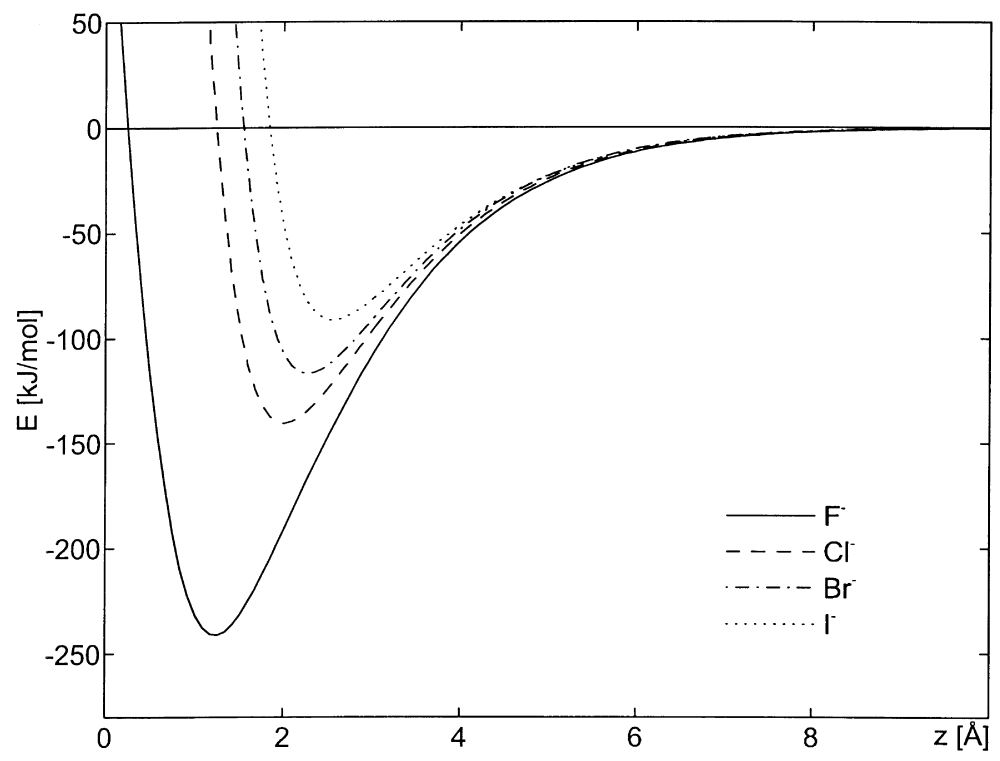

Fig. 3. The behaviour of the potential $V_{2}$, when the ions are adsorbed at the hollow site of the $\mathrm{Cu}(100)$ surface. 


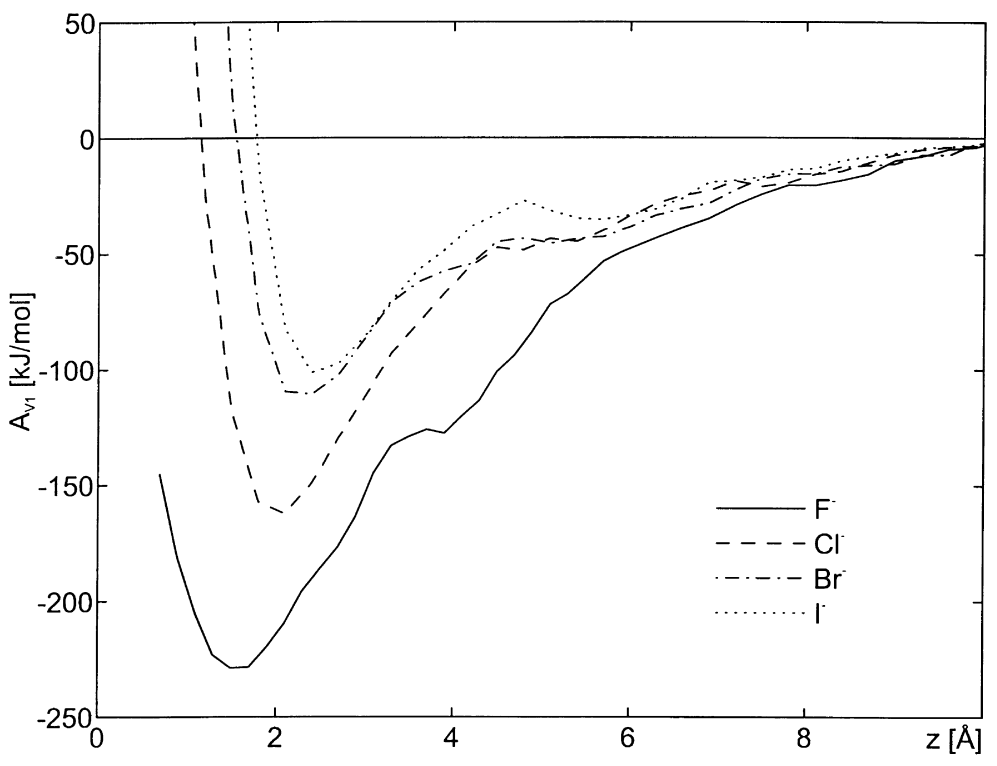

Fig. 4. The total mean force on the halide ions as they approach the $\mathrm{Cu}(100)$ surface. These curves are obtained as a combination of the solvent mean force on the ion, $A^{\mathrm{s}}$, with the ion-metal potential $V_{1}$.

Two extreme approaches were tested for the interpretation of the cluster model calculations as a starting point for the construction of the ion-metal interaction. In one of them, $V_{1}$, the cluster has been treated as a very limited approximation of the electrode, what is expressed by building this potential as a combination of the ion-atom interactions. When summed over twelve $\mathrm{Cu}$ atoms, the quantum results are reconstructed; when summed over all 300 atoms forming the metal wall in the simulation it gives the total

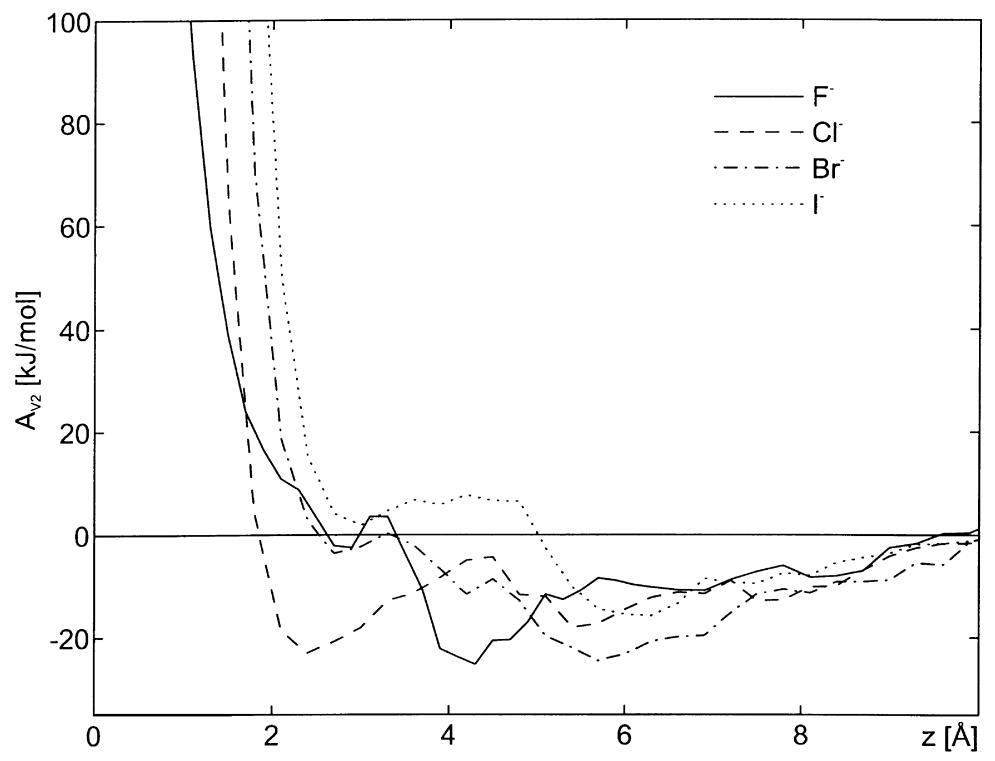

Fig. 5. As in Fig. 4, but the curves are obtained as a combination of the solvent mean force on the ion with the ion-metal potential $V_{2}$. 
ion-wall interaction. The behaviour of this potential is shown in Fig. 2 for the adsorption of ions at the hollow site of the $\mathrm{Cu}(100)$ surface. In the second approach, the results of the cluster calculations have been treated as exact estimates of the total interaction of the ion with the wall. This potential, $V_{2}$, simply reconstructs the quantum points and is shown in Fig. 3 for the adsorption of the ions at the hollow site of the $\mathrm{Cu}(100)$ wall.

When the summation runs only over the atoms belonging to the cluster used in the quantum calculations, both potentials can be said to behave almost identically but their properties differ significantly when they are applied to the infinite wall. The reason for this large difference lies in the long range interactions as described by potential $V_{1}$ where even very distant atoms participate to some non-negligible extent into the total ion-metal interaction. Since the ion-cluster optimal interaction energy is characterized by a quite large value at the optimal position of the ion, the long range interaction is still non-zero even at the distances from the surface of more than $10 \AA$.

The results of combining the ion-wall interaction estimated by these two approaches with the solvent mean force presented in Fig. 1 is shown in Fig. 4 for the potential $V_{1}$ and in Fig. 5 for the potential $V_{2}$. It can be seen that the way of defining the ion-metal interaction is indeed crucial for the final description of the specific adsorption phenomenon. In the first case, as in earlier theoretical works [27], all ions are found to be specifically adsorbed. The strongest adsorption is predicted by this model for fluoride, and the weakest for iodide. One should notice that, if only one ion, iodide, is considered to be described by this approximation, the free energy of adsorption appears to be close to the experimental estimates of this property. The free energy of adsorption of halide ions on mercury and silver surfaces has been reported to be of about -80 to $-100 \mathrm{~kJ} \mathrm{~mol}^{-1}$, depending on the ion. Nevertheless, when the results for other ions are considered, this model appears to be incorrect.

It can be seen in Fig. 5, that the second approach to the ion-metal interaction is also not satisfactory. The ion-copper interaction is relatively weak in this approximation and is not able to compensate the repulsive response of the solvent when ions come closer to the surface. Nevertheless, an important feature should be noticed at the same time. The tendencies observed on these curves do agree qualitatively with experimental reports. For the three larger ions two minima can be found on the free energy curves, corresponding to the non-specific and to the contact adsorption. For fluoride, the curve of the potential of mean force exhibits only one minimum and becomes repulsive in the region where the ion would be specifically adsorbed.

Another problem which comes from the nature of the quantum calculations is the description of the interaction with metal when the ion is located in the solution, at a certain distance from the surface. It has been shown [76] that the description of this long range interaction by the classical model of the image charge potential is also quite ambiguous, especially at distances closer to the metal. This is due to the uncertain definition of the location of the image plane in relation to the real corrugated structure of the metal surface. In fact, it was shown that the free energy curve obtained with the ion-metal interaction as expressed by the image charge potential is determined by the position if the image plane.

We have been looking at some factors that may influence the potential derived from the cluster model calculations. In this paper we discuss especially those related to the problem of the construction of the potential, leaving the question of the adequacy of the cluster model calculations to be analyzed elsewhere.

First of all, it is not easy to decide about the type of potential to be used. As mentioned above, the additive potential commonly used in the literature, may not give the best description of the interaction of the ion with an electrode. In this approach all atoms of the electrode are equivalent from the point of view of the ion, while this is very unlikely to be true in reality. The interaction of the ion with an atom belonging to the surface layer of the metal is expected to be different from that with an atom at the same distance from the ion, but located deep inside the metal. Subtle effects such as screening by other atoms as well as the non-uniform charge distribution inside the metal slab will certainly perturb the ion-atom interaction in its usual interpretation. If the additive approximation were essentially correct, it should be enough to calculate just the ion-atom interaction, construct the simple potential by fitting some analytical function to these results and then make the summation over the larger aggregate of the metal atoms. Such 


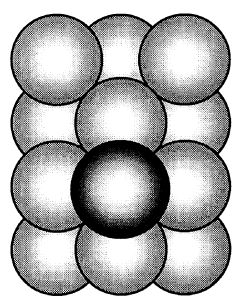

$\mathrm{Cu}_{12}-\mathrm{F}$

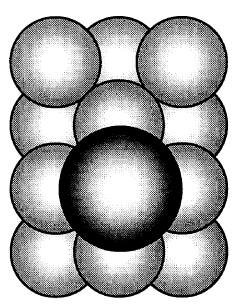

$\mathrm{Cu}_{12}-\mathrm{Cl}^{-}$

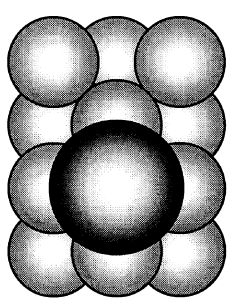

$\mathrm{Cu}_{12}-\mathrm{Br}$

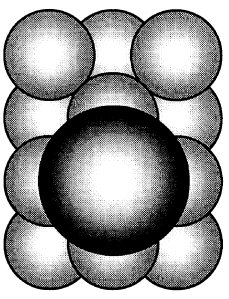

$\mathrm{Cu}_{12-\mathrm{I}}$

Fig. 6. The realistic drawing of the $\mathrm{Cu}_{12}-\mathrm{X}$ clusters using the same scale for the radii of the copper atoms forming the cluster and of the four halide ions.

summations made over atoms forming the cluster used in the quantum calculations should then reconstruct the computed results. Unfortunately, this is not true, since the interaction of the ion with a single metal atom is different from that with this same atom involved in metal-metal interactions. This effect of the environmental differentiation of metal atoms should be in some way taken into account by the potential used.

On the other hand, accepting that the simple additive potential is not correct, the alternative of using direct ion-metal cluster interaction should be critically evaluated in function of the size of the ion relatively to the metal cluster used. When talking about the interaction of the ion with the metal one usually has in mind the simple model of the ion as a dimensionless point charge. Of course, the potential includes the size of the species studied only through its dependency on the distances if it is a pairwise additive ion-metal atom potential. If not, the range of the specific interaction between the ion and atoms belonging to its closest neighbourhood should be in some way defined, being in a certain way a function of the size of the ion. Nevertheless, in some cases it may be not enough, especially when the potential is based on the cluster model calculations. It should be noticed that when comparative studies are performed, the size of the metal cluster usually stays the same in all calculations, while the size of the ion adsorbed on its surface differs significantly. In Fig. 6 the four $\mathrm{Cu}_{12}$ $\mathrm{X}^{-}$clusters $(\mathrm{X}=\mathrm{F}, \mathrm{Cl}, \mathrm{Br}, \mathrm{I})$ are drawn taking into account the relation between the atomic radius of copper atoms and the ionic radii of the halide ions. It can be seen that the $\mathrm{Cu}_{12}$ cluster used in DFT calculations is relatively large comparing to the size of fluoride, but it is rather small for iodide. This demonstrates that, in the approximation used, the model of the metal surface is not the same from the point of view of the ion. Iodide, as a very large ion, almost reaches the edges of the cluster and so 'sees' the $\mathrm{Cu}_{12}$ cluster as a very limited area of the surface and an important part of the ion-wall interaction is missing. For the relatively small fluoride, the cluster used may be said to be large enough to approximate the surface and so for this ion the results of the $\mathrm{Cu}_{12}-\mathrm{F}^{-}$quantum calculations are probably much closer to the total ion-wall interaction than for the other three ions. The difficulty with this line of reasoning is that this 'size error' effect is hard to evaluate quantitatively.

Another problem in the construction of the ionmetal interaction, independently of the additive or non-additive potential used, is its adequacy for the description of the intermediate region, when the ion approaching the surface begins to have its first solvation shell modified. This problem has been mentioned in some earlier works, but no attempt was made to include this parameter into the form of the potential used. The quantum calculations describe the interaction of the ions or other species with the metal in vacuum. Of course, this model is true only in a very limited range of distances of the particle to the surface. When the interaction of the ion with the electrode is considered at the water-metal interface the quantum calculations are not valid any more, since some water molecules may be located between the ion and the metal, modifying significantly the character of the ion-electrode interaction. It is obvious that 
Table 1

The upper section shows the optimal ion-metal distance, $z_{\min }$, as estimated by DFT calculations of the $\mathrm{Cu}_{12}-\mathrm{X}^{-}$system and the solvent contribution to the potential of mean force, $A^{\mathrm{s}}$, at that distance. The lowest section shows the total potential of mean force, A, on the ions at the $z_{\min }$ distance or, in some cases, at the specific optimal distance (shown in parentheses). This is obtained as a combination of $A^{\mathrm{s}}$ with the metal-ion interaction energy, $E$, obtained in the models $V_{\mathrm{im}}, V_{\mathrm{im} 0}, V_{1}, V_{2}$ and $V_{3}$ discussed in the text

\begin{tabular}{lrrrr}
\hline Propertylion & \multicolumn{1}{l}{$\mathrm{F}^{-}$} & $\mathrm{Cl}^{-}$ & $\mathrm{Br}^{-}$ & $\mathrm{I}^{-}$ \\
\hline$Z_{\min }$ & 1.3 & 2.0 & 2.3 & 2.6 \\
$A^{\mathrm{s}}$ & 300.1 & 126.9 & 122.3 & 97.4 \\
$E_{\mathrm{im}}$ & $-2448(1.3)$ & $-390(2.0)$ & $-273(2.3)$ & $-208(2.6)$ \\
$E_{\mathrm{im} 0}$ & $-217(1.3)$ & $-134(2.0)$ & $-130(2.3)$ & $-98(2.6)$ \\
$E_{\mathrm{V} 1}$ & $-539(1.0)$ & $-296(1.8)$ & $-242(2.1)$ & $-206(2.4)$ \\
$E_{\mathrm{V} 2}$ & $-242(1.3)$ & $-142(2.0)$ & $-118(2.3)$ & $-93(2.6)$ \\
$E_{\mathrm{V} 3}$ & $-346(1.1)$ & $-232(1.8)$ & $-151(2.3)$ & $-183(2.4)$ \\
$A_{\mathrm{im}}$ & $-2148(1.3)$ & $-264(2.0)$ & $-111(2.3)$ & $-111(2.6)$ \\
$A_{\mathrm{im} 0}$ & $84(1.3)$ & $-162(2.0)$ & $-0.8(2.6)$ \\
$A_{\mathrm{V} 1}$ & $-229(1.5)$ & $-11(2.0)$ & $-72(2.3)$ & $-101(2.4)$ \\
$A_{\mathrm{V} 2}$ & $60(1.3)$ & $-104(2.1)$ & $7(2.6)$ \\
$A_{\mathrm{V} 3}$ & $-52(1.7)$ & & $-79(2.4)$ \\
\hline
\end{tabular}

the quantum points give a very poor approximation when the ion is located in the bulk of the liquid, far away from the electrode. In this region the image charge potential is likely to give a better description of the long range electrostatic forces between the ion and the electrode.

A new ion-metal potential, $V_{3}$, where some of the factors discussed above are taken into account is being tested right now. Special attention is given to a more accurate evaluation of the interaction of the ion with the wall in the intermediate region where this is assumed to be a mixture of the image charge potential with a short-range additive potential. The range of this specific ion-metal interaction has been defined to be dependent on the size of the ion in relation to the size of the cluster used in the DFT calculations. The intermediate region, where this specific interaction is smoothly replaced by the image charge potential, is determined by the size of the first hydration shell of each ion and the range of the first hydration layer of the metal. In a certain region both types of ion-metal interaction, specific and non-specific, are assumed to participate in the total ion-metal potential.

In Table 1 some preliminary results obtained with this new potential are given. The optimal energy of adsorption of the ion on the metal surface in a vacuum, as well as the free energy of adsorption of each ion on the $\mathrm{Cu}(100)$ surface are given. For comparison, the analogous results obtained with the $V_{1}$ and $V_{2}$ potentials, and with two extreme interpretations of the image charge potential, $V_{\mathrm{im}}$ and $V_{\text {im0 }}$, are also given. In the $V_{\mathrm{im} 0}$ potential, the image plane is defined by the centers of atoms belonging to the first metal layer. In the alternative $V_{\mathrm{im}}$, this plane is shifted towards the liquid by a distance of $1.28 \AA$. Additionally, the values of the solvent contribution, $A^{\mathrm{s}}$, at the distances predicted for the adsorption of the ions in the DFT calculations are listed.

Several tendencies in the results presented in this table should be stressed. First, all potentials tested do follow the same ordering in the strength of the ioncopper interaction as that found from the DFT calculations, i.e. $\mathrm{F}^{-}>\mathrm{Cl}^{-}>\mathrm{Br}^{-}>\mathrm{I}^{-}$. Nevertheless, the energy values differ significantly when different models are compared and so do the free energy values. As mentioned earlier, in the case of the image charge potential, the results are very sensitive to the position of the image plane. The $A_{\mathrm{V} 1}$ value for iodide is close to the experimental estimate, but an increasing error is observed when going to fluoride along the halide ions group. The tendencies found in the $A_{\mathrm{V} 2}$ free energies follow the experimental suggestions in a qualitative way, but cannot be compared quantitatively.

The preliminary results obtained with the potential $V_{3}$ appear to be very promising, when compared with those obtained by the standard models. The $A_{\mathrm{V} 3}$ estimates of the free energy of adsorption of ions are close to the values proposed experimentally for the adsorption of the halide ions on noble metals [36]. The weakest adsorption is that of fluoride as predicted by this 
model when a comparison is made with the three other ions and this agrees with values reported from the electrochemical measurements. A somewhat unclear situation is found for chloride, that is predicted to be more strongly adsorbed than iodide. This deviation can be associated with some assumptions made about the strength of the interaction of this ion with the water molecule.

\section{Conclusions}

It is a well known fact, that the interactions at the electrode-electrolyte interface are very difficult to measure experimentally. As shown above, the theoretical evaluation of the forces between species belonging to these two phases is not a trivial problem and depends on many factors. The description of the interfacial interaction is loaded with many doubts and ambiguities. Several problems associated with the construction of the potentials describing the interfacial interactions have been pointed out. Most of them are related to the cluster model calculations that are the usual starting point in studies of this type. The additivity of the potential assumed in the simulations to describe the ion-metal interaction seems to be oversimplifying the problem. Some aspects of the accuracy of the cluster model as a basis for the fitting of the potential have been discussed.

The correction of some problems analyzed above is included in a new potential being tested. The preliminary results obtained with this potential do confirm that more sophisticated models than those used in the past for the ion-metal interaction are necessary to succeed in the study of the subtle balance of forces acting on the ion near the electrode. In the new model proposed the ion-metal interaction increases when going from iodide to fluoride following the tendency found from the DFT cluster calculations. Nevertheless, when combined with the solvent mean force acting on the ion near the surface, the adsorptivity of fluoride is the smallest in agreement with the common experimental wisdom. At the same time the computed values of free energy of adsorption of the three larger ions appear to be close to the level found experimentally for this property.

\section{Acknowledgements}

The project discussed here originated from discussions with Prof. S. Romanowski of the University of Lodz (Poland) and we thank him for his suggestions. The financial support of Praxis XXI through project PRAXIS/2/2.1/QUI/474/94 is acknowledged. A.I. thanks PRAXIS XXI for a doctoral scholarship.

\section{References}

[1] S.L. Carnie, Ber. Bunsenges. Phys. Chem. 91 (1987) 262.

[2] C.Y. Lee, J.A. McCammon, P.J. Rossky, J. Chem. Phys. 80 (1984) 4448.

[3] N.G. Parsonage, D. Nicholson, J. Chem. Soc., Faraday Trans. 82 (1986) 1521.

[4] N.G. Parsonage, D. Nicholson, J. Chem. Soc., Faraday Trans. 83 (1987) 663.

[5] J.P. Valleau, A. Gardner, J. Chem. Phys. 86 (1987) 4162.

[6] A. Gardner, J.P. Valleau, J. Chem. Phys. 86 (1987) 4171.

[7] E. Spohr, K. Heinzinger, Ber. Bunsenges. Phys. Chem. 92 (1988) 1358.

[8] K. Foster, K. Raghavan, M. Berkowitz, Chem. Phys. Lett. 162 (1989) 32.

[9] E. Spohr, J. Phys. Chem. 93 (1989) 6171.

[10] K. Heinzinger, E. Spohr, Electrochim. Acta 34 (1989) 1849.

[11] J.I. Siepmann, M. Sprik, J. Chem. Phys. 102 (1995) 511.

[12] G. Nagy, K. Heinzinger, J. Electroanal. Chem. 296 (1990) 549.

[13] K. Heinzinger, Pure Appl. Chem. 63 (1991) 1733.

[14] K. Raghavan, K. Foster, K. Motakabir, M. Berkowitz, J. Chem. Phys. 94 (1991) 2110.

[15] J. Böcker, Z. Gurskii, K. Heinzinger, J. Phys. Chem. 100 (1996) 14969.

[16] K.J. Schweighofer, X. Xia, M.L. Berkowitz, Langmuir 12 (1996) 3749.

[17] D.A. Rose, I. Benjamin, J. Chem. Phys. 98 (1993) 2283.

[18] J. Seitz-Beywl, M. Poxleitner, K. Heinzinger, Z. Naturforsch. 46a (1991) 876.

[19] J.N. Glosli, M.R. Philpott, J. Chem. Phys. 96 (1992) 6962.

[20] J.N. Glosli, M.R. Philpott, J. Chem. Phys. 98 (1993) 9995.

[21] J.N. Glosli, M.R. Philpott, Electrochim. Acta. 41 (1996) 2145.

[22] G. Tóth, K. Heinzinger, Chem. Phys. Lett. 245 (1995) 49.

[23] E. Spohr, G. Tóth, K. Heinzinger, Electrochim. Acta 41 (1996) 2131.

[24] D.A. Rose, I. Benjamin, J. Chem. Phys. 95 (1991) 6856.

[25] L. Perrera, M.L. Berkowitz, J. Phys. Chem 97 (1993) 13803.

[26] E. Spohr, Chem. Phys. Lett. 207 (1993) 214.

[27] E. Spohr, Acta. Chem. Scand. 49 (1995) 189.

[28] R.R. Nazmutdinov, E. Spohr, J. Phys. Chem. 98 (1994) 5956.

[29] A.R. Newmark, W. Schmickler, J. Electroanal. Chem. 329 (1992) 159.

[30] O. Pecina, W. Schmickler, E. Spohr, J. Electroanal. Chem. 394 (1995) 29. 
[31] O. Pecina, W. Schmickler, E. Spohr, J. Electroanal. Chem. 405 (1996) 239.

[32] A. Hamelin, T. Vitanov, E. Sevastyanov, A. Popov, J. Electroanal. Chem. 145 (1983) 225.

[33] R. Parsons, Chem. Rev. 90 (1990) 813.

[34] R. Payne, Trans. Faraday Soc. 64 (1968) 1638.

[35] G.J. Hills, R.M. Reeves, J. Electroanal. Chem. 42 (1973) 355.

[36] D. Larkin, K.L. Guyer, J.T. Hupp, M.J. Weaver, J. Electroanal. Chem. 138 (1982) 401.

[37] M. Weidenauer, K.G. Weil, Ber. Bunsenges. Phys. Chem. 92 (1988) 1368.

[38] J.O’M. Bockris, M. Gamboa-Aldeco, M. Szklarczyk, J. Electroanal. Chem. 339 (1992) 355.

[39] H.D. Hurwitz, A. Jenard, B. Bicamumpaka, W. Schmickler, J. Electroanal. Chem. 349 (1993) 49.

[40] B.M. Jovic, V.D. Jovic, D.M. Drazic, J. Electroanal. Chem. 399 (1995) 197.

[41] X. Gao, M.J. Weaver, J. Am. Chem. Soc. 114 (1992) 8544.

[42] W. Haiss, J.K. Sass, X. Gao, M.J. Weaver, Surf. Sci. Lett. 274 (1992) L593.

[43] X. Gao, M.J. Weaver, J. Phys. Chem. 97 (1993) 8685.

[44] X. Gao, M.J. Weaver, Ber. Bunsenges. Phys. Chem. 97 (1993) 507.

[45] X. Gao, G.J. Edens, M.J. Weaver, J. Phys. Chem. 98 (1994) 8074.

[46] X. Gao, G.J. Edens, F.C. Liu, A. Hamelin, M.J. Weaver, J. Phys. Chem. 98 (1994) 8086.

[47] D.W. Suggs, A.J. Bard, J. Phys. Chem. 99 (1995) 8349.

[48] T. Yamada, N. Batina, K. Itaya, J. Phys. Chem. 99 (1995) 8817.

[49] J. Wang, B.M. Ocko, A.J. Davenport, H.S. Isaacs, Phys. Rev. B46 (1992) 10321.

[50] B.M. Ocko, G.M. Watson, J. Wang, J. Phys. Chem. 98 (1994) 897.

[51] O.M. Magnussen, B.M. Ocko, R.R. Adzic, J.X. Wang, Phys. Rev. B51 (1995) 5510.

[52] H. Wetzel, H. Gerischer, B. Pettinger, Chem. Phys. Lett. 207 (1993) 455.

[53] P. Gao, M.J. Weather, J. Phys. Chem. 90 (1986) 4057.

[54] B. Pettinger, M.R. Philpott, J.G. Gordon II, J. Phys. Chem. 85 (1981) 2746.
[55] G. Niaura, A. Malinauskas, Chem. Phys. Lett. 207 (1993) 455.

[56] A. Ignaczak, J.A.N.F. Gomes, Chem. Phys. Lett. 257 (1996) 609.

[57] A. Ignaczak, J.A.N.F. Gomes, J. Electroanal. Chem. 420 (1997) 71.

[58] A. Ignaczak, J.A.N.F. Gomes, J. Electroanal. Chem. 420 (1997) 209.

[59] C. Au, J. Breza, M.W. Roberts, Chem. Phys. Lett. 66 (1979) 340.

[60] B.A. Sexton, A.E. Hughes, Surf. Sci. 140 (1984) 227.

[61] M.A. Leban, A.T. Hubbard, J. Electroanal. Chem. 74 (1976) 253.

[62] D. Döhnert, J. Koutecky, J.W. Shultze, J. Electroanal. Chem. 82 (1977) 81.

[63] F. Illas, F. Sanz, J. Virgili, J. Electroanal. Chem. 142 (1982) 31.

[64] M. Weissmann, N.V. Cohan, J. Electroanal. Chem. 146 (1983) 171.

[65] M. Jauregui, N.V. Cohan, M. Weissmann, J. Electroanal. Chem. 163 (1984) 381.

[66] G.V. Kulkarni, S.K. Rangarajan, J. Electroanal. Chem. 196 (1985) 375

[67] An. Kuznetsov, J. Reinhold, W. Lorentz, J. Electroanal. Chem. 164 (1984) 167.

[68] An. Kuznetsov, J. Reinhold, W. Lorentz, Electrochim. Acta. 29 (1984) 801.

[69] An. Kuznetsov, R.R. Nazmutdinov, R.R. Shapnik, Electrokhim. 22 (1986) 776.

[70] M. Blanco, J. Rubio, F. Illas, J. Electroanal. Chem. 261 (1989) 39.

[71] M. Blanco, J.M. Ricart, J. Rubio, F. Illas, J. Electroanal. Chem. 267 (1989) 243.

[72] An. Kuznetsov, Electrochim. Acta. 40 (1995) 2483.

[73] J. Seitz-Beywl, M. Poxleitner, M.M. Probst, K. Heinzinger, Int. J. Quant. Chem 42 (1992) 1141.

[74] G. Tóth, E. Spohr, K. Heinzinger, Chem. Phys. 200 (1995) 347.

[75] G. Pacchioni, Electrochim. Acta 41 (1996) 2285.

[76] A. Ignaczak, J.A.N.F. Gomes, S. Romanowski, J. Electroanal. Chem. 450 (1998) 175. 